\title{
Exact charged black-hole solutions in D-dimensional $f(T)$ gravity: torsion vs curvature analysis
}

\section{S. Capozziello, ${ }^{a, b}$ P.A. González, ${ }^{c}$ E.N. Saridakis ${ }^{d, e, f}$ and Y. Vásquez ${ }^{g}$}

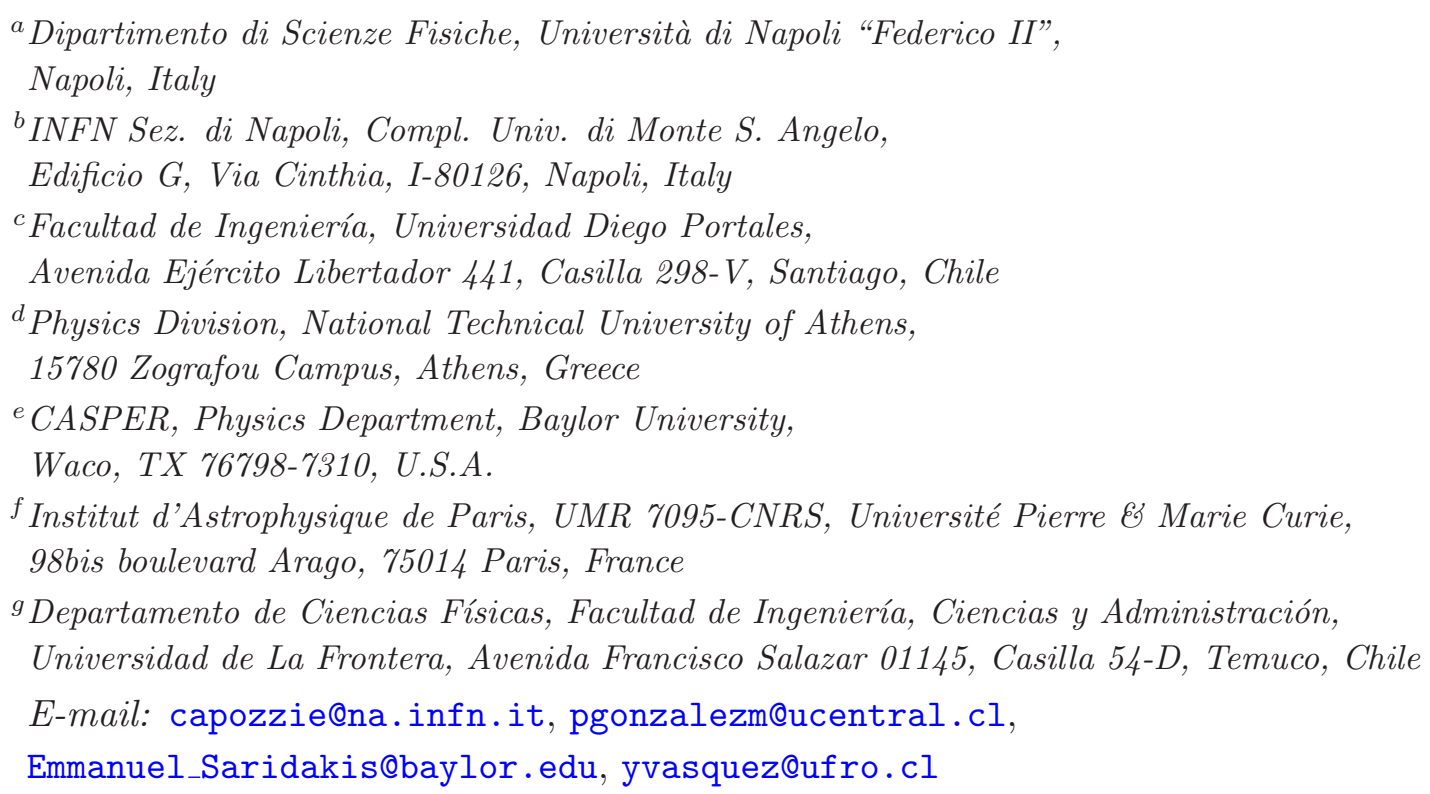

ABSTRACT: We extract exact charged black-hole solutions with flat transverse sections in the framework of D-dimensional Maxwell- $f(T)$ gravity, and we analyze the singularities and horizons based on both torsion and curvature invariants. Interestingly enough, we find that in some particular solution subclasses there appear more singularities in the curvature scalars than in the torsion ones. This difference disappears in the uncharged case, or in the case where $f(T)$ gravity becomes the usual linear-in- $T$ teleparallel gravity, that is General Relativity. Curvature and torsion invariants behave very differently when matter fields are present, and thus $f(R)$ gravity and $f(T)$ gravity exhibit different features and cannot be directly re-casted each other.

Keywords: Black Holes, Spacetime Singularities, Classical Theories of Gravity

ARXIV EPRINT: 1210.1098 


\section{Contents}

$\begin{array}{llr}1 & \text { Introduction } & 1\end{array}$

2 Teleparallel Equivalent to General Relativity and its $f(T)$ extension 2

3 D-dimensional teleparallel gravity and its Maxwell- $f(T)$ extension $\quad 3$

4 Exact charged solutions $\quad 5$

$\begin{array}{ll}4.1 \text { Radial electric field } & 7\end{array}$

4.2 Zero radial field 9

4.3 Magnetic field and radial electric field 9

$\begin{array}{lll}4.4 & \text { Schwarzschild and Reissner-Nordström solutions } & 10\end{array}$

5 Singularities and horizons $\quad 11$

$\begin{array}{llr}6 & \text { Concluding remarks } & 18\end{array}$

\section{Introduction}

Teleparallel equivalent of General Relativity (TEGR) $[1,2]$ is an equivalent formulation of gravity, but, instead of using curvature invariants defined by the Levi-Civita connection, the Weitzenböck connection is adopted. Therefore TEGR exhibits no curvature but only torsion. The dynamical objects in such a framework are the four linearly independent vierbeins and the advantage of this framework is that the torsion tensor is formed solely by products of first derivatives of these vierbeins. In such a formulation, as described in [2], the Lagrangian density, $T$, can then be constructed from this torsion tensor assuming the invariance under general coordinate transformations, global Lorentz transformations, and the parity operation, along with demanding the Lagrangian density to be second order in the torsion tensor. In [3-6] an extension of the above idea was constructed, making the Lagrangian density a function of $T$, similar to the $f(R)$ extension of the Hilbert-Einstein action. $f(T)$ gravity has gained a significant attention in the literature, and proves to exhibit interesting cosmological implications [3-45].

Such an approach can be framed within the class of new gravity theories aimed to extend General Relativity in order to solve its shortcomings at Infra-Red and Ultra-Violet scales [46]. Clearly, in extending the geometry sector, one of the goals is to solve the puzzle of dark energy and dark matter that, up to now, seems to have no counterpart at fundamental level. In other words, both $f(T)$ gravity and $f(R)$ gravity could be reliable approaches to address the problems of missing matter and accelerated expansion without asking for new material ingredients that have not been detected yet by the experiments [47]. 
In this work we investigate D-dimensional $f(T)$ gravity, considering additionally the electromagnetic sector. Exact black-hole solutions with flat transverse section (BanadosTeitelboim-Zanelli (BTZ)-like solutions [48]) can be derived for a given range of parameter space. Then we analyze the singularities of these solutions based on the torsion scalar and the curvature scalar, pointing out differences with respect to $f(R)$ gravity. It is important to stress that searching for exact solutions is a fundamental step to set a new field theory. Exact solutions allow a full control of the systems and can contribute to the well-formulation and well-position of the Cauchy problem (for a discussion on this point see [49]).

The paper is organized as follows. In section 2, we present a brief review of TEGR in four dimensions, as well as of its $f(T)$ extension. In section 3 the D-dimensional teleparallel gravity is formulated and the analysis is extended to D-dimensional Maxwell- $f(T)$ gravity. In section 4 we derive exact charged static solutions and section 5 is devoted to the investigation of singularities and horizons. Finally, in section 6 we discuss some physical implications of the results.

\section{Teleparallel Equivalent to General Relativity and its $f(T)$ extension}

In this section we briefly review Teleparallel Equivalent to General Relativity (TEGR) in four dimensions and its $f(T)$ extension. Throughout the manuscript we use the following notation: greek indices $\mu, \nu, \ldots$ run over all space-time coordinates $0,1,2,3$; lower case Latin indices (from the middle of the alphabet) $i, j, \ldots$ run over spatial coordinates 1,2 , 3 ; capital Latin indices $A, B, \ldots$ run over the tangent space-time $0,1,2,3$, and lower case Latin indices (from the beginning of the alphabet) $a, b, \ldots$ run over the tangent space spatial coordinates $1,2,3$.

As we mentioned above, the dynamical variable of teleparallel gravity is the vierbein field $\mathbf{e}_{A}\left(x^{\mu}\right)$, which forms an orthonormal basis for the tangent space at each point $x^{\mu}$ of the manifold, that is $\mathbf{e}_{A} \cdot \mathbf{e}_{B}=\eta_{A B}$, with $\eta_{A B}=\operatorname{diag}(1,-1,-1,-1)$. Moreover, the vector $\mathbf{e}_{A}$ can be expressed in terms of its components $e_{A}^{\mu}$ in a coordinate basis, namely $\mathbf{e}_{A}=e_{A}^{\mu} \partial_{\mu}$. In such a formulation the metric tensor is acquired from the dual vierbein as

$$
g_{\mu \nu}(x)=\eta_{A B} e_{\mu}^{A}(x) e_{\nu}^{B}(x)
$$

Although in General Relativity one uses the torsionless Levi-Civita connection, in the present construction one uses the curvatureless Weitzenböck connection [50], whose torsion tensor reads

$$
T_{\mu \nu}^{\lambda}=\stackrel{\mathrm{w}}{\Gamma}_{\nu \mu}^{\lambda}-\stackrel{\mathrm{w}}{\Gamma}_{\mu \nu}^{\lambda}=e_{A}^{\lambda}\left(\partial_{\mu} e_{\nu}^{A}-\partial_{\nu} e_{\mu}^{A}\right) .
$$

Moreover, the contorsion tensor, which gives the difference between Weitzenböck and LeviCivita connections, is given by $K_{\rho}^{\mu \nu}=-\frac{1}{2}\left(T_{\rho}^{\mu \nu}-T_{\rho}^{\nu \mu}-T_{\rho}{ }^{\mu \nu}\right)$, while it proves convenient to define $S_{\rho}{ }^{\mu \nu}=\frac{1}{2}\left(K_{\rho}^{\mu \nu}+\delta_{\rho}^{\mu} T_{\alpha}^{\alpha \nu}-\delta_{\rho}^{\nu} T_{\alpha}^{\alpha \mu}\right)$. For a detailed exposition of torsion tensor properties see [51].

In conclusion, in the present formulation the torsion tensor $T_{\mu \nu}^{\lambda}$ includes all the information concerning the gravitational field. Using the above definitions one can construct the 
simplest form of the "teleparallel" Lagrangian, which is the torsion scalar, that is $[52,53]$

$$
\mathcal{L}=T \equiv \frac{1}{4} T^{\rho \mu \nu} T_{\rho \mu \nu}+\frac{1}{2} T^{\rho \mu \nu} T_{\nu \mu \rho}-T_{\rho \mu}{ }^{\rho} T^{\nu \mu}{ }_{\nu} .
$$

Thus, the simplest action of teleparallel gravity reads:

$$
S=\frac{1}{2 \kappa} \int d^{4} x e\left(T+\mathcal{L}_{m}\right),
$$

where $\kappa=8 \pi G, e=\operatorname{det}\left(e_{\mu}^{A}\right)=\sqrt{-g}$ and $\mathcal{L}_{m}$ accounts for the matter Lagrangian. It is worth noticing here that the Ricci scalar $R$ and the torsion scalar $T$ differ only by a total derivative of the torsion tensor, namely [54]:

$$
R=-T-2 \nabla^{\mu}\left(T_{\mu \nu}^{\nu}\right) .
$$

Varying the action (2.4) with respect to the vierbein we obtain the field equations

$$
e^{-1} \partial_{\mu}\left(e e_{A}^{\rho} S_{\rho}^{\mu \nu}\right)-e_{A}^{\lambda} T^{\rho}{ }_{\mu \lambda} S_{\rho}^{\nu \mu}-\frac{1}{4} e_{A}^{\nu} T=4 \pi G e_{A}^{\rho}{ }^{\mathrm{em}}{ }_{\rho}^{\nu},
$$

where the tensor $\stackrel{\text { em }}{T} \rho_{\rho}^{\nu}$ on the right-hand side is the usual energy-momentum tensor of matter fields. These equations coincide with those of General Relativity for every geometry choice, and this is the why the theory is named "Teleparallel Equivalent to General Relativity".

One can generalize the above formulation considering arbitrary functions of the torsion scalar $f(T)$ in the gravitational action [3-6], although the Lorentz invariance of the linear theory seems to be spoiled [55-57]. Thus, the action becomes

$$
S=\frac{1}{2 \kappa} \int d^{4} x e\left[T+f(T)+\mathcal{L}_{m}\right] .
$$

Notice the difference in the various conventions in $4 \mathrm{D}-f(T)$ literature, since some authors replace $T$ by $f(T)$, while the majority replace $T$ by $T+f(T)$. In this work we follow the second convention, that is teleparallel gravity is acquired by setting $f(T)=0$. Finally, variation of the action (2.7) with respect to the vierbein gives the field equations

$e^{-1} \partial_{\mu}\left(e e_{A}^{\rho} S_{\rho}^{\mu \nu}\right)\left(1+\frac{d f}{d T}\right)-e_{A}^{\lambda} T^{\rho}{ }_{\mu \lambda} S_{\rho}{ }^{\nu \mu}+e_{A}^{\rho} S_{\rho}{ }^{\mu \nu} \partial_{\mu}(T) \frac{d^{2} f}{d T^{2}}-\frac{1}{4} e_{A}^{\nu}[T+f(T)]=4 \pi G e_{A}^{\rho} \stackrel{\text { em }}{T}_{\rho}^{\nu}$.

\section{D-dimensional teleparallel gravity and its Maxwell- $f(T)$ extension}

In this section we present teleparallel gravity in D-dimensions and its Maxwell- $f(T)$ extension and we explore its properties. It proves more convenient to use differential forms, where the torsion 2-form $T^{a}$ is simply $T^{a}=d e^{a}$.

We start with the gravitational teleparallel action with the most general quadratic form in the torsion tensor. Under the assumption of zero spin-connection it is given by $[58,59]$

$$
S=\frac{1}{2 \kappa} \int\left(\rho_{0} \mathcal{L}_{0}+\rho_{1} \mathcal{L}_{1}+\rho_{2} \mathcal{L}_{2}+\rho_{3} \mathcal{L}_{3}+\rho_{4} \mathcal{L}_{4}\right),
$$


where $\rho_{i}$ are dimensionless parameters and

$$
\begin{array}{lll}
\mathcal{L}_{0}=\frac{1}{4} e^{a} \wedge \star e_{a}, & \mathcal{L}_{1}=d e^{a} \wedge \star d e_{a}, & \mathcal{L}_{2}=\left(d e_{a} \wedge e^{a}\right) \wedge \star\left(d e_{b} \wedge e^{b}\right), \\
\mathcal{L}_{3}=\left(d e^{a} \wedge e^{b}\right) \wedge \star\left(d e_{a} \wedge e_{b}\right), & \mathcal{L}_{4}=\left(d e_{a} \wedge e^{b}\right) \wedge \star\left(d e_{b} \wedge e^{a}\right),
\end{array}
$$

with $\star$ standing for the Hodge dual operator and $\wedge$ for the usual wedge product. The coupling constant $\rho_{0}=-\frac{8}{3} \Lambda$ accounts for the cosmological constant term, and furthermore, since $\mathcal{L}_{3}$ can be completely expressed in terms of $\mathcal{L}_{1}$, in the following we set $\rho_{3}=0$ [58]. Lastly, we mention that in the above expression $\kappa$ is the D-dimensional gravitational constant, while the vierbeins and the metric are now D-dimensional. Therefore, in the following, all the conventions adopted in section 2 extend in $\mathrm{D}$ dimensions.

Action (3.1) can be written more conveniently as

$$
S=\frac{1}{2 \kappa} \int(T-2 \Lambda) \star 1=\frac{1}{2 \kappa} \int d^{D} x e(T-2 \Lambda),
$$

where $\star 1=(-1)^{D-1} e^{0} \wedge e^{1} \wedge e^{2} \ldots \ldots \wedge e^{D-1}$, and the torsion scalar $T$ is given by

$$
T=(-1)^{D-1} \star\left[\rho_{1}\left(d e^{a} \wedge \star d e_{a}\right)+\rho_{2}\left(d e_{a} \wedge e^{a}\right) \wedge \star\left(d e_{b} \wedge e^{b}\right)+\rho_{4}\left(d e_{a} \wedge e^{b}\right) \wedge \star\left(d e_{b} \wedge e^{a}\right)\right] .
$$

Expanding this expression in its components we acquire

$$
T=\frac{1}{2}\left(\rho_{1}+\rho_{2}+\rho_{4}\right) T^{a b c} T_{a b c}+\rho_{2} T^{a b c} T_{b c a}-\rho_{4} T_{a}^{a c} T_{b c}^{b},
$$

thus we straightforwardly see that for $\rho_{1}=0, \rho_{2}=-\frac{1}{2}$ and $\rho_{4}=1$ it coincides with (2.3) in $\mathrm{D}$ dimensions, namely

$$
T=\frac{1}{4} T^{a b c} T_{a b c}-\frac{1}{2} T^{a b c} T_{b c a}-T_{a}^{a c} T_{b c}^{b} .
$$

Now, we will extend the above discussion considering arbitrary functions of the torsion scalar $f(T)$ in the D-dimensional gravitational action. Thus, we consider an action of the form

$$
S=\frac{1}{2 \kappa} \int d^{D} x e[T+f(T)-2 \Lambda]
$$

with the torsion scalar $T$ given by (3.5), that is we keep the general coefficients $\rho_{i}$. In differential forms the above action can be written as

$$
S=\frac{1}{2 \kappa} \int\{[f(T)+T-2 \Lambda] \star 1\},
$$

where now $T$ is given by (3.4). Finally, note that teleparallel D-dimensional gravity discussed above is obtained by setting $f(T)=0$.

Lastly, we extend the discussion incorporating additionally the electromagnetic sector. In particular, we extend the total action to

$$
S=\frac{1}{2 \kappa} \int\{[f(T)+T-2 \Lambda] \star 1\}+\int \mathcal{L}_{F},
$$


where

$$
\mathcal{L}_{F}=-\frac{1}{2} F \wedge^{\star} F
$$

is the Maxwell Lagrangian, while $F=d A$, with $A \equiv A_{\mu} d x^{\mu}$, is the electromagnetic potential 1-form. The action variation leads to the following field equations:

$$
\begin{aligned}
\delta \mathcal{L}=\delta e^{a} \wedge & \left\{( 1 + \frac { d f } { d T } ) \left\{\rho_{1}\left[2 d \star d e_{a}+i_{a}\left(d e^{b} \wedge \star d e_{b}\right)-2 i_{a}\left(d e^{b}\right) \wedge \star d e_{b}\right]\right.\right. \\
& +\rho_{2}\left\{-2 e_{a} \wedge d \star\left(d e^{b} \wedge e_{b}\right)+2 d e_{a} \wedge \star\left(d e^{b} \wedge e_{b}\right)+i_{a}\left[d e^{c} \wedge e_{c} \wedge \star\left(d e^{b} \wedge e_{b}\right)\right]\right. \\
& \left.\quad-2 i_{a}\left(d e^{b}\right) \wedge e_{b} \wedge \star\left(d e^{c} \wedge e_{c}\right)\right\} \\
& +\rho_{4}\left\{-2 e_{b} \wedge d \star\left(e_{a} \wedge d e^{b}\right)+2 d e_{b} \wedge \star\left(e_{a} \wedge d e^{b}\right)\right. \\
& \left.\left.+i_{a}\left[e_{c} \wedge d e^{b} \wedge \star\left(d e^{c} \wedge e_{b}\right)\right]-2 i_{a}\left(d e^{b}\right) \wedge e_{c} \wedge \star\left(d e^{c} \wedge e_{b}\right)\right\}\right\} \\
+ & 2 \frac{d^{2} f}{d T^{2}} d T\left[\rho_{1} \star d e_{a}+\rho_{2} e_{a} \wedge \star\left(d e_{b} \wedge e^{b}\right)+\rho_{4} e_{b} \wedge \star\left(d e^{b} \wedge e_{a}\right)\right] \\
& \left.+\left[f(T)-T \frac{d f}{d T}\right] \wedge \star e_{a}-2 \Lambda \star e_{a}-\frac{1}{2}\left[F \wedge i_{a}(\star F)-i_{a}(F) \wedge \star F\right]\right\} \\
& +\delta A\left(d^{\star} F\right)=0,
\end{aligned}
$$

where $i_{a}$ is the interior product. Although one could investigate solution subclasses with general coupling parameters $\rho_{i}$, in the following, for the sake of simplicity, we restrict to the standard case $\rho_{1}=0, \rho_{2}=-1 / 2$ and $\rho_{4}=1$ of (3.6).

\section{Exact charged solutions}

Let us now investigate the charged solutions of the theory. In order to extract the static solutions we consider the metric form

$$
d s^{2}=F(r)^{2} d t^{2}-\frac{1}{G(r)^{2}} d r^{2}-r^{2} \sum_{i=1}^{i=D-2} d x_{i}^{2},
$$

which arises from the vierbein diagonal ansatz

$$
e^{0}=F(r) d t, \quad e^{1}=\frac{1}{G(r)} d r, \quad e^{2}=r d x_{1}, \quad e^{3}=r d x_{2}, \quad \ldots
$$

Let us make an important comment here concerning the vierbein choice that corresponds to the metric (4.1). In the case of linear-in- $T$ gravity the above simple, diagonal relation between the metric (4.1) and the vierbeins (4.2) is always allowed. On the contrary, in the extension of $f(T)$ gravity, in general, one could have a more complicated relation connecting the vierbein with the metric, with the vierbein being non-diagonal even for a diagonal metric [60-63]. However, in the cosmological investigations of $f(T)$ gravity [345], as well as in its black-hole solutions [64-68], the authors still use the diagonal relation between the vierbeins and the metric, as a first approach to reveal the features of the theory. Thus, in the present investigation we also impose the diagonal relation between 
the vierbeins and the metric, as a first approach on the subject and in order to reveal the main features of the solution structure. However, we are aware that a detailed study of the general vierbein choice (and its relation to extra degrees of freedom) is a necessary step for a deeper understanding of $f(T)$-gravity foundations.

Concerning the electric sector of the electromagnetic 2-form we assume

$$
F=d A=E_{r}(r) e^{1} \wedge e^{0}+\sum_{i=1}^{i=D-2} E_{i}(r) e^{0} \wedge e^{i+1},
$$

where $E_{r}$ is the radial electric field, neglecting for the moment the magnetic part. Thus, inserting the above ansatzes in the field equations (3.11), we finally obtain

$$
\begin{gathered}
\left(1+\frac{d f}{d T}\right) T-\left[f(T)-T \frac{d f}{d T}\right]+2 \Lambda+\frac{1}{2} E_{r}^{2}-\frac{1}{2} \sum_{i=1}^{i=D-2} E_{i}^{2}=0, \\
\left(1+\frac{d f}{d T}\right)\left[-\frac{G(r) G^{\prime}(r)}{r}+\frac{F^{\prime}(r) G(r)^{2}}{r F(r)}\right]-\frac{d^{2} f}{d T^{2}} T^{\prime}(r) \frac{G(r)^{2}}{r}-\frac{1}{2} \sum_{i=1}^{i=D-2} E_{i}^{2}=0 \\
\left(1+\frac{d f}{d T}\right)\left[-\frac{F^{\prime \prime}(r) G(r)^{2}}{F(r)}-\frac{F^{\prime}(r) G^{\prime}(r) G(r)}{F(r)}+\frac{F^{\prime}(r) G(r)^{2}}{r F(r)}\right] \\
-\left(1+\frac{d f}{d T}\right)\left[(D-3) \frac{G^{\prime}(r) G(r)}{r}-(D-3) \frac{G(r)^{2}}{r^{2}}\right] \\
-\frac{d^{2} f}{d T^{2}} T^{\prime}(r)\left[\frac{F^{\prime}(r) G(r)^{2}}{F(r)}+(D-3) \frac{G(r)^{2}}{r}\right]+\frac{1}{2} E_{r}^{2}-\frac{1}{2} E_{1}^{2}=0, \\
E_{r} E_{j}=0 \quad j=1, \ldots, D-2, \\
E_{i} E_{j}=0 \quad i, j=1, \ldots, D-2(i \neq j),
\end{gathered}
$$

where

$$
T(r)=2(D-2) \frac{F^{\prime}(r) G(r)^{2}}{r F(r)}+(D-2)(D-3) \frac{G(r)^{2}}{r^{2}} .
$$

The remaining field equations are equivalent to equation (4.6), that is the $a=j$ equation is similar to (4.6), but with $-\frac{1}{2} E_{1}^{2}$ replaced by $-\frac{1}{2} E_{j-1}^{2}$.

A first observation is that from (4.4) we deduce that $T$ has, in general, an $r$-dependence, which disappears for a zero electric charge. Such a behavior reveals the new features that are brought in by the richer structure of the addition of the electromagnetic sector. Moreover, form (4.7) and (4.8), we deduce that we cannot have simultaneously two non-zero electric field components. This result is similar to the known no-go theorem of 3D GR-like gravity $[69,70]$, which states that configurations with two non-vanishing components of the Maxwell field are dynamically not allowed. However, it is not valid anymore if we add the magnetic sector, as we will see in subsection 4.3 (it holds only for $\mathrm{D}=3$ ). Therefore, in the following we investigate the cases of radial electric field, of non-radial electric field, and of magnetic and radial electric field, separately. 


\subsection{Radial electric field}

We first consider the case where there exists only radial electric field. Thus, the Maxwell equations give

$$
E_{r}=\frac{Q}{r^{D-2}},
$$

where $Q$ is an integration constant which, as usual, coincides with the electric charge of the black hole. Now, integrating equation (4.5) we find the very simple and helpful result

$$
F(r)=G(r)\left(1+\frac{d f}{d T}\right)
$$

Using equations (4.11) and (4.9) we obtain

$$
\frac{d G(r)^{2}}{d r}+\left[2 \frac{d}{d r} \ln \left(1+\frac{d f}{d T}\right)+\frac{(D-3)}{r}\right] G(r)^{2}-\frac{r T(r)}{(D-2)}=0
$$

whose solution is

$$
G(r)^{2}=\frac{1}{\left(1+\frac{d f}{d T}\right)^{2} r^{D-3}}\left[\frac{1}{(D-2)} \int\left(1+\frac{d f}{d T}\right)^{2} r^{D-2} T(r) d r+\text { Const }\right]
$$

and using equation (4.11) we get

$$
F(r)^{2}=\frac{1}{r^{D-3}}\left[\frac{1}{(D-2)} \int\left(1+\frac{d f}{d T}\right)^{2} r^{D-2} T(r) d r+\text { Const }\right],
$$

where Const is an integration constant related to the mass of the spherical object.

In order to proceed, and similar to [68], we will consider Ultraviolet (UV) corrections of $f(T)$ gravity. In particular, we examine the modifications on the solutions caused by UV modifications of D-dimensional gravity and we consider a representative ansatz of the form $f(T)=\alpha T^{2}$. This is the first order correction in every realistic $f(T)$ gravity, in which we expect $f(T) \ll T[14,37]$, since $T$ (like $R$ ) is small in $\kappa^{2}$-units. Thus, for $\alpha \neq 0$, equation (4.4) leads to

$$
T(r)=\frac{-1 \pm \sqrt{1-24 \alpha \Lambda-6 \alpha Q^{2} r^{4-2 D}}}{6 \alpha}
$$

with the upper and lower signs corresponding to the positive and negative branch solutions respectively (note that if $\alpha=0$ then (4.4) becomes linear having only one solution, which is given by the $\alpha \rightarrow 0$ limit of the positive branch of (4.15), namely $T(r)=-Q^{2} r^{4-2 D} / 2-2 \Lambda$, in which case teleparallel gravity is restored). Thus,

$$
1+\frac{d f}{d T}=\frac{2}{3} \pm \frac{1}{3} \sqrt{1-24 \alpha \Lambda-6 \alpha Q^{2} r^{4-2 D}}
$$


and therefore performing the integration that appears in (4.13) and (4.14), we obtain

$$
\begin{aligned}
& \int\left(1+\frac{d f}{d T}\right)^{2} r^{D-2} T(r) d r=\frac{1}{54 \alpha}\left[-\frac{18 \alpha Q^{2} r^{3-D}}{3-D}-\frac{(1+72 \alpha \Lambda) r^{D-1}}{D-1}\right] \\
& \pm \frac{\sqrt{r^{4 D}\left(1-24 \alpha \Lambda-6 \alpha Q^{2} r^{4-2 D}\right)}}{54 \alpha}\left[\frac{6 \alpha Q^{2} r^{3-3 D}}{2 D-5}-\frac{(-1+24 \alpha \Lambda) r^{-1-D}}{D-1}\right] \\
& \mp \frac{(D-2)^{2}(-1+24 \alpha \Lambda) Q^{2} r^{3+D} \sqrt{1+\frac{6 \alpha Q^{2} r^{4-2 D}}{-1+24 \alpha \Lambda}}{ }_{2} F_{1}\left(\frac{D-3}{2(D-2)}, \frac{1}{2}, \frac{3 D-7}{2(D-2)} ; \frac{6 \alpha Q^{2} r^{4-2 D}}{1-24 \alpha \Lambda}\right)}{3(D-3)(2 D-5)(D-1) \sqrt{r^{4 D}\left(1-24 \alpha \Lambda-6 \alpha Q^{2} r^{4-2 D}\right)}},
\end{aligned}
$$

where ${ }_{2} F_{1}(a, b, c ; x)$ is the hypergeometric function. We mention that the last argument of this function, namely $\left(6 \alpha Q^{2} r^{4-2 D}\right) /(1-24 \alpha \Lambda)$, must be negative, while from (4.15) it is required that $1-24 \alpha \Lambda-6 \alpha Q^{2} r^{4-2 D}$ must be positive, therefore we deduce that $\alpha$ should be negative.

In summary, inserting the integral (4.17), along with (4.16), in (4.13) and (4.14), we find that the black-hole solution is:

$$
\begin{aligned}
& G(r)^{2}=\frac{1}{\left(\frac{2}{3} \pm \frac{1}{3} \sqrt{1-24 \alpha \Lambda-6 \alpha Q^{2} r^{4-2 D}}\right)^{2} r^{D-3}}\left\{\frac{1}{(D-2)}\right. \\
& \left\{\frac{1}{54 \alpha}\left[-\frac{18 \alpha Q^{2} r^{3-D}}{3-D}-\frac{(1+72 \alpha \Lambda) r^{D-1}}{D-1}\right]\right. \\
& \pm \frac{\sqrt{r^{4 D}\left(1-24 \alpha \Lambda-6 \alpha Q^{2} r^{4-2 D}\right)}}{54 \alpha}\left[\frac{6 \alpha Q^{2} r^{3-3 D}}{2 D-5}-\frac{(-1+24 \alpha \Lambda) r^{-1-D}}{D-1}\right] \\
& \left.\mp \frac{(D-2)^{2}(-1+24 \alpha \Lambda) Q^{2} r^{3+D} \sqrt{1+\frac{6 \alpha Q^{2} r^{4-2 D}}{-1+24 \alpha \Lambda}}{ }_{2} F_{1}\left(\frac{D-3}{2(D-2)}, \frac{1}{2}, \frac{3 D-7}{2(D-2)} ; \frac{6 \alpha Q^{2} r^{4-2 D}}{1-24 \alpha \Lambda}\right)}{3(D-3)(2 D-5)(D-1) \sqrt{r^{4 D}\left(1-24 \alpha \Lambda-6 \alpha Q^{2} r^{4-2 D}\right)}}\right\} \\
& + \text { Const }\}
\end{aligned}
$$

and

$$
\begin{aligned}
& F(r)^{2}=\frac{1}{r^{D-3}}\left\{\frac{1}{(D-2)}\right. \\
& \left\{\frac{1}{54 \alpha}\left[-\frac{18 \alpha Q^{2} r^{3-D}}{3-D}-\frac{(1+72 \alpha \Lambda) r^{D-1}}{D-1}\right]\right. \\
& \pm \frac{\sqrt{r^{4 D}\left(1-24 \alpha \Lambda-6 \alpha Q^{2} r^{4-2 D}\right)}}{54 \alpha}\left[\frac{6 \alpha Q^{2} r^{3-3 D}}{2 D-5}-\frac{(-1+24 \alpha \Lambda) r^{-1-D}}{D-1}\right] \\
& \left.\mp \frac{(D-2)^{2}(-1+24 \alpha \Lambda) Q^{2} r^{3+D} \sqrt{1+\frac{6 \alpha Q^{2} r^{4-2 D}}{-1+24 \alpha \Lambda}}{ }_{2} F_{1}\left(\frac{D-3}{2(D-2)}, \frac{1}{2}, \frac{3 D-7}{2(D-2)} ; \frac{6 \alpha Q^{2} r^{4-2 D}}{1-24 \alpha \Lambda}\right)}{3(D-3)(2 D-5)(D-1) \sqrt{r^{4 D}\left(1-24 \alpha \Lambda-6 \alpha Q^{2} r^{4-2 D}\right)}}\right\} \\
& + \text { Const }\} .
\end{aligned}
$$


The special point in the parameter space $\Lambda=1 /(24 \alpha)$ needs to be analyzed separately, since in this point we obtain the solution

$$
\int\left(1+\frac{d f}{d T}\right)^{2} r^{D-2} T(r) d r=-\frac{r^{-1-5 D}}{27 \alpha}\left[\frac{2 r^{6 D}}{D-1}-\frac{9 \alpha Q^{2} r^{4+4 D}}{D-3} \pm \frac{3 \sqrt{6}\left(-\alpha Q^{2} r^{4+2 D}\right)^{3 / 2}}{2 D-5}\right]
$$

and thus

$$
\begin{aligned}
& G(r)^{2}=\frac{1}{\left(\frac{2}{3} \pm \frac{1}{3} \sqrt{\left.1-24 \alpha \Lambda-6 \alpha Q^{2} r^{4-2 D}\right)^{2} r^{D-3}}\left\{\frac{1}{(D-2)}\right.\right.} \\
& \left.\left\{-\frac{r^{-1-5 D}}{27 \alpha}\left[\frac{2 r^{6 D}}{D-1}-\frac{9 \alpha Q^{2} r^{4+4 D}}{D-3} \pm \frac{3 \sqrt{6}\left(-\alpha Q^{2} r^{4+2 D}\right)^{3 / 2}}{2 D-5}\right]\right\}+\text { Const }\right\}
\end{aligned}
$$

and

$$
\begin{aligned}
& F(r)^{2}=\frac{1}{r^{D-3}}\left\{\frac{1}{(D-2)}\right. \\
& \left.\left\{-\frac{r^{-1-5 D}}{27 \alpha}\left[\frac{2 r^{6 D}}{D-1}-\frac{9 \alpha Q^{2} r^{4+4 D}}{D-3} \pm \frac{3 \sqrt{6}\left(-\alpha Q^{2} r^{4+2 D}\right)^{3 / 2}}{2 D-5}\right]\right\}+\text { Const }\right\} .
\end{aligned}
$$

Finally, the case $D=3$ has to be analyzed separately. Taking properly the limit $D=3$ of the above expressions we obtain the solutions extracted in [68] for 3D Maxwell$f(T)$ gravity. Lastly, one can straightforwardly check that in the limit $\alpha \rightarrow 0$ (of the positive branch since in this case the negative branch disappears) one re-obtains the usual charged General Relativity solutions.

\subsection{Zero radial field}

Let us for the moment assume that we have zero radial field. In this case equation (4.8) implies that we can have at most one non-zero component of the electric field along the non-radial (transversal) directions. However, as we mentioned below equation (4.9), for $D>3$ the remaining field equations are similar to equation (4.6) but with $-\frac{1}{2} E_{1}^{2}$ replaced by $-\frac{1}{2} E_{j-1}^{2}$, therefore subtracting these equations we acquire the conditions $E_{i}^{2}=E_{j}^{2}$, with $i$ and $j$ running from 1 to $D-2$. These conditions, along with equation (4.8), yield $E_{i}=0$ $(i=1, \ldots, D-2)$ for $D>3$, that is the electric field is completely zero. The only cases where zero radial electric field does not lead to a disappearance of the total electric field is for $D=3$ (where a non-zero azimuthal electric field is possible) which was analyzed in detail in [68], or if we consider simultaneously non-zero non-radial electric field with magnetic field, case which lies beyond the scope of the present investigation.

\subsection{Magnetic field and radial electric field}

For completeness we also examine the case where magnetic field is present. While in $D=3$ we deduce that electric field must be absent [68], for $D>3$ one can simultaneously have non-zero magnetic and electric fields. As an explicit example we consider an electromagnetic strength 2-form in four dimensions given by

$$
F=E_{r}(r) e^{1} \wedge e^{0}+B_{23}(r) e^{2} \wedge e^{3},
$$


that is we consider a radial electric field $E_{r}$ and a magnetic field $B_{23}$ both depending on the radial coordinate $r$ only. From the Maxwell equations in four dimensions for the electric field we immediately obtain

$$
E_{r}(r)=\frac{Q}{r^{2}},
$$

while incorporating the equations of motion analogous to (4.4)-(4.8) we can see that a solution is obtained by

$$
B_{23}(r)=\frac{P}{r^{2}},
$$

leading to the metric coefficients (4.19) and (4.20) with $Q^{2}+P^{2}$ in place of $Q^{2}$ (and for $D=4)$.

\subsection{Schwarzschild and Reissner-Nordström solutions}

We close this section with some comments on the black-hole solution structure of $f(T)$ gravity. By definition, every solution of TEGR is also a solution of $f(T)$ gravity, for the special case $f(T)=0$. In principle, a non-trivial $f(T)$ ansatz, that is a non-trivial correction to TEGR, will give rise to non-trivial corrections to the solutions of TEGR, that is to the solutions of General Relativity. This was analyzed in detail in the above subsections.

However, one could follow a different approach and ask what should be the specific $f(T)$ ansatz in order to obtain exactly the same solutions with TEGR. Thus, in the following we extract the $f(T)$ form in order for the theory to accept as exact solutions the usual Reissner-Nordström or Schwarzschild black holes. In this case we impose the diagonal vierbeins corresponding to the metric (for completeness we allow also for a general transverse curvature and for a cosmological constant)

$$
d s^{2}=G(r)^{2} d t^{2}-\frac{1}{G(r)^{2}} d r^{2}-r^{2} d \Omega_{k},
$$

with

$$
G(r)^{2}=k-\frac{2 M}{r}+\frac{Q^{2}}{r^{2}}-\frac{\Lambda}{3} r^{2},
$$

where $k=1,-1,0$ represents the curvature of the transverse section, corresponding to a spherical, hyperbolic or plane section respectively. Concerning the electric sector of the electromagnetic 2-form we assume

$$
F=d A=E_{r}(r) e^{1} \wedge e^{0},
$$

where $E_{r}$ is the radial electric field. Inserting the above ansatzes in the field equations (3.11) we obtain

$$
\begin{aligned}
\left(1+\frac{d f}{d T}\right) T-\left[f(T)-T \frac{d f}{d T}\right]+2 \Lambda+\frac{1}{2} E_{r}^{2} & =0, \\
\frac{d^{2} f}{d T^{2}} T^{\prime}(r) & =0, \\
\left(1+\frac{d f}{d T}\right)\left[-G^{\prime \prime}(r) G(r)-G^{\prime}(r)^{2}+\frac{G(r)^{2}}{r^{2}}\right]+\frac{1}{2} E_{r}^{2} & =0, \\
T(r)=4 \frac{G^{\prime}(r) G(r)}{r}+2 \frac{G(r)^{2}}{r^{2}} & =-\frac{2 Q^{2}}{r^{4}}+\frac{2 k}{r^{2}}-2 \Lambda .
\end{aligned}
$$


From (4.30) we obtain that either $\frac{d^{2} f}{d T^{2}}=0$, in which case we re-obtain TEGR, or $T^{\prime}(r)=0$, in which case (4.32) and then (4.29) and (4.31) lead to $k=0, Q=0$ and $f(T)=\alpha \sqrt{T}$, with $\alpha$ an integration constant. Therefore, we conclude that there is no $f(T)$ ansatz that can lead to the usual charged black-hole solution (Reissner-Nordström -de Sitter black hole) of TEGR. However, there is indeed a specific $f(T)$ ansatz, namely $f(T)=\alpha \sqrt{T}$, that leads to the Schwarzschild-de Sitter - like solution with flat transverse section. This is similar to the corresponding analysis of $f(R)$ gravity, where one finds that although a general $f(R)$ form leads to corrections in the black-hole solutions of General Relativity [71, 72], there is a specific form, namely $f(R)=\alpha \sqrt{R+\beta}$, which leads exactly to the Schwarzschild solution [73, 74] (however note that in $f(R)$ case the solution is exactly Schwarzschild, and not Schwarzschild-like with flat transverse section).

\section{$5 \quad$ Singularities and horizons}

Let us now investigate the singularities and the horizons of the above general solutions. The first step is to find at which $r$ do the functions $G(r)^{2}$ and $F(r)^{2}$ become zero or infinity. However, since these singularities may correspond to coordinate singularities, the usual procedure is to investigate various invariants, since if these invariants diverge at one point they will do that independently of the specific coordinate basis, and thus the corresponding point is a physical singularity (note that the opposite is not true, that is the finiteness of an invariant is not a proof that there is not a physical singularity there). In standard black-hole literature of curvature-formulated gravity (either General Relativity or its modifications), one usually studies the Ricci scalar, the Kretschmann scalar, or other invariants constructed by the Riemann tensor and its contractions.

In teleparallel description of gravity, one has, in principle, two approaches of finding invariants. The first is to use the solution for the vierbein and the Weitzenböck connection in order to calculate torsion invariants such as the torsion scalar $T$. The second is to use the solution for the corresponding metric in order to construct the Levi-Civita connection, and then use it to calculate curvature invariants such are the Ricci and Kretschmann scalars (a calculation of curvature scalars using straightaway the Weitzenböck connection leads to zero by construction). The comparison of both approaches is a main subject of interest of the present work, capable of pointing out differences between curvature and torsion gravity.

In particular, we are going to investigate whether one can formulate everything in terms of vierbens, Weitzenböck's connection and torsion invariants, as one can do with the metric, the Levi-Civita connection and curvature invariants. Perhaps one could say that the use of curvature invariants, instead of torsion ones, is better justified by the fact that in a realistic theory matter is coupled to the gravitational sector through the metric and not through the vierbeins (with the interesting exception of fermionic matter), and particles follow geodesics defined by the Levi-Civita connection. On the other hand, one could say that the two approaches are equivalent only with a suitable, non-diagonal, relation between the vierbeins and the metric. In any case, while at the classical level the above approaches could look equivalent or alternative, for the quantization procedure it would be crucial 
to determine whether the metric or the vierbein is the fundamental field. Therefore, the following analysis can enlighten this subject.

The torsion invariant $T$, that is the torsion scalar, that arises from the vierbein solution (4.2) with the use of Weitzenböck's connection is (4.9), which in the examined case becomes just (4.15). The curvature invariants that arise from the metric solution (4.1) through the calculation of the Levi-Civita connection are

$$
\begin{aligned}
R= & -2 \frac{G(r)^{2} F^{\prime \prime}(r)}{F(r)}-2 \frac{G(r) G^{\prime}(r) F^{\prime}(r)}{F(r)}-2(D-2) \frac{G(r)^{2} F^{\prime}(r)}{r F(r)} \\
& -2(D-2) \frac{G(r) G^{\prime}(r)}{r}-(D-2)(D-3) \frac{G(r)^{2}}{r^{2}}, \\
R_{\mu \nu} R^{\mu \nu}= & \frac{G(r)^{2}}{F(r)^{2} r^{2}}\left[r F^{\prime \prime}(r) G(r)+r F^{\prime}(r) G^{\prime}(r)+(D-2) G(r) F^{\prime}(r)\right]^{2} \\
& +\frac{G(r)^{2}}{F(r)^{2} r^{2}}\left[r F^{\prime \prime}(r) G(r)+r F^{\prime}(r) G^{\prime}(r)+(D-2) G^{\prime}(r) F(r)\right]^{2} \\
& +(D-2) \frac{G(r)^{2}}{F(r)^{2} r^{4}}\left[r G(r) F^{\prime}(r)+r G^{\prime}(r) F(r)+(D-3) G(r) F(r)\right]^{2}, \\
R_{\mu \nu \rho \sigma} R^{\mu \nu \rho \sigma}= & 4 \frac{G(r)^{2}}{F(r)^{2}}\left[F^{\prime \prime}(r) G(r)+F^{\prime}(r) G^{\prime}(r)\right]^{2}+4(D-2) \frac{G(r)^{4} F^{\prime}(r)^{2}}{r^{2} F(r)^{2}} \\
& +4(D-2) \frac{G^{\prime}(r)^{2} G(r)^{2}}{r^{2}}+2(D-2)(D-3) \frac{G(r)^{4}}{r^{4}},
\end{aligned}
$$

being respectively the Ricci scalar, the Ricci tensor square and the Kretschmann scalar. Note that, using (4.9), the Ricci scalar is given by

$$
R=-T-2 \frac{G(r)^{2} F^{\prime \prime}(r)}{F(r)}-2 \frac{G(r) G^{\prime}(r) F^{\prime}(r)}{F(r)}-2(D-2) \frac{G(r) G^{\prime}(r)}{r},
$$

which is just relation (2.5) calculated for the vierbeins (4.2).

Observing the form of the torsion scalar $T$ in (4.15) we deduce that in the charged case it diverges only at $r=0$. This can be alternatively verified examining the form (4.9) along with the expressions (4.18), (4.19) (or (4.21), (4.22) for the special solution).

Observing the forms of Ricci and Kretschmann scalars in (5.1), (5.3) we deduce that in the charged case the possible divergence points are at $r=0$, at the points where $G(r)^{2} \rightarrow \infty$, or at the roots of $F(r)$. From the solutions for $G(r)$ and $F(r)$ of (4.18), (4.19) we straightforwardly obtain that $r=0$ indeed leads to divergent Ricci and Kretschmann scalars. From the form of $G(r)$ in (4.18) along with (4.16) we observe that $G(r)^{2} \rightarrow \infty$ at $1+\frac{d f}{d T}=0$, that is at

$$
r_{s}=\left(-\frac{1+8 \alpha \Lambda}{2 \alpha Q^{2}}\right)^{\frac{1}{4-2 D}},
$$

a relation which holds only for the negative branch, since for the positive branch $1+\frac{d f}{d T}$ has no roots (additionally since $\alpha<0$ the above solution is real only for $\Lambda<-1 /(8 \alpha)$ ). Indeed 
one can straightforwardly see that the Ricci and Kretschmann scalars do diverge at $r=r_{s}$. Finally, concerning the roots of $F(r)$, due to (4.11), namely $F(r)=G(r)\left(1+\frac{d f}{d T}\right)$, they are just the roots of $G(r)$, since $F(r)$ remains finite and non-zero at $1+\frac{d f}{d T}=0$ since at this point $G(r)^{2} \rightarrow \infty$. Taking the corresponding limits and using (4.18), (4.19), we can see that the roots of $G(r)$ leads always to finite Ricci and Kretschmann scalars. All the above hold also for the special solution (4.21), (4.22). In summary, in the charged case the Ricci and Kretschmann scalars diverge at $r=0$ and at $r=r_{s}$ given by (5.4). Lastly, we mention that in the uncharged case the Ricci scalar vanishes, however the Kretschmann scalar behaves as in the charged case.

From the above analysis we are led to the very interesting result that in some cases the singularities obtained by the torsion scalar analysis are less than those obtained by the curvature scalar analysis. In particular, this happens for the negative branch of the solutions, for $Q \neq 0$ and for $\Lambda<-1 /(8 \alpha)$, in which case the curvature invariants possess an additional physical singularity at $r=r_{s}$ given by (5.4). We stress that when $f(T)=0$, that is in the case of usual teleparallel gravity, the negative branch disappears as we mentioned above, thus the singularity analyses of the two approaches coincide. Additionally, in the uncharged case, that is when $Q=0$, the extra singularity at $r_{s}$ disappears too, and the singularity analyses of the two approaches coincide too. In conclusion, we deduce that the above difference in the physical singularities of the torsion and curvature analysis, is a result of both the non-linear $f(T)$ structure and of the non-zero electric charge, which reveals the novel features that are brought in in the theory in this case.

Let us discuss on the horizons of the above solutions. Although we showed that the roots of $G(r)$ at $r>0$ (if they exist) do not correspond to physical singularities, obviously they correspond to horizons, since they appear in the denominator in the metric (4.1) and in the vierbeins (4.2). In order to show that the roots of $G(r)$, say at $r=r_{H}$, are just coordinate singularities, we consider the Painlevé-Gullstrand coordinates [75-78] through the transformation $d t=d \tau+g(r) d r$, with $g(r)$ a function of the radial coordinate. Therefore, the metric (4.1) becomes

$$
d s^{2}=F(r)^{2} d \tau^{2}+2 g(r) F(r)^{2} d r d \tau-\left[\frac{1}{G(r)^{2}}-F(r)^{2} g(r)^{2}\right] d r^{2}-r^{2} \sum_{i=1}^{i=D-2} d x_{i}^{2} .
$$

Choosing $g(r)^{2}=\frac{1}{F(r)^{2}}\left[\frac{1}{G(r)^{2}}-1\right]$ and defining

$$
h(r)^{2}=F(r)^{2} / G(r)^{2}=\left[2+\sqrt{1-24 \alpha \Lambda-6 \alpha Q^{2} r^{4-2 D}}\right] / 3
$$

we can bring it in a flat Euclidean form

$$
d s^{2}=F(r)^{2} d \tau^{2}+2 h(r) \sqrt{1-G(r)^{2}} d r d \tau-d r^{2}-r^{2} \sum_{i=1}^{i=D-2} d x_{i}^{2},
$$

which is regular at $r=r_{H}$. Therefore, $r=r_{H}$, if they exist, are just coordinate singularities, that is horizons. 
From the above analysis it is implied that the black-hole solutions of the charged $f(T)$ gravity may possess a horizon at $r_{H}$ that shields the physical singularities. However, firstly it is not guaranteed that $r_{H}$ exists, since there could be parameter choices for which $G(r)$ has no roots, that is the physical singularity at $r=0$ becomes naked. Secondly, even if $r_{H}$ exists it is not guaranteed that it will shield the second physical singularity of the charged negative branch at $r=r_{s}$ given by (5.4), since this will depend on the specific parameter choice. In particular, we can see that if $F\left(r_{s}\right)^{2}<0$ then $r_{H}$ exists and shields the singularity at $r_{s}$, that is $r_{H}>r_{s}$, otherwise $r_{s}$ is a naked singularity. This is not the case for $f(T) \rightarrow 0$ or $Q \rightarrow 0$, in which, as we mentioned, $r_{s}$ disappears. Therefore, we conclude that the cosmic censorship theorem, namely that there are always horizons that shield the physical singularities, does not always hold for $f(T)$ gravity, a result that was already found in the $3 \mathrm{D}$ case too [68].

Before proceeding to the numerical elaboration of the obtained solutions, we make the following comment. In curvature gravity there can be cases where the Ricci scalar is finite at one point although there is a physical singularity there, which is revealed through the use of the Kretschmann scalar, and that is why people usually examine both scalars simultaneously. Thus, one could ask whether one should use additional torsion scalars too, defined as various contractions of the torsion tensor. In particular, according to (2.3), the torsion scalar $T$ contains three separate scalars, corresponding to different contractions of the torsion tensor, namely $I_{1}=T^{\mu \nu \rho} T_{\mu \nu \rho}, I_{2}=T^{\rho \mu \nu} T_{\nu \mu \rho}, I_{3}=T_{\rho \mu}{ }^{\rho} T^{\nu \mu}{ }_{\nu}$, and thus one could additionally examine their behavior in order to reveal the singularities. However, it is well known that these separate combinations are not invariant under local Lorentz transformations, and that was the reason that the teleparallel Lagrangian (torsion scalar) $T$ was defined as their specific combination which becomes Lorentz invariant [2]. Therefore, one cannot use other torsion scalars apart from $T$ in order to investigate the singularities (the explicit calculation of $I_{i}$ 's for the obtained vierbein solutions shows that they acquire different values in different coordinates, and thus they are not invariants, however their specific combination in $T$ does acquire the same value independently of the coordinate basis and thus it is a well-defined invariant).

In order to provide a more transparent picture of the above singularity and horizon behavior, we proceed to the numerical elaboration of specific examples. Since the asymptotic behavior of $G(r)$ is given by

$$
G(r)^{2}=-\Lambda_{\mathrm{eff}} r^{2}+\ldots,
$$

where ... correspond to sub-leading terms and

$$
\Lambda_{\mathrm{eff}}=\frac{9}{54 \alpha(D-1)(D-2)(2 \pm \sqrt{1-24 \alpha \Lambda})}\left[1+72 \alpha \Lambda \mp(1-24 \alpha \Lambda)^{3 / 2}\right],
$$

we can distinguish three subclasses, namely the asymptotically AdS one $\left(\Lambda_{\text {eff }}<0\right)$, the asymptotically dS one $\left(\Lambda_{\text {eff }}>0\right)$ and the limiting $\Lambda_{\text {eff }}=0$ one. Note that the second and third solution subclasses can never be obtained by the negative solution branch. Without loss of generality we consider $D=4, \alpha=-1$ and Const $=-1$, while we suitably choose $\Lambda$ in order to lie in the above three subclasses, which we investigate separately. 
- Case $\Lambda_{\text {eff }}<0$

Let us consider $\Lambda=-1 / 25$, which satisfies the condition $\Lambda_{\text {eff }}<0$. In figure 1 from up to down we depict $G(r)^{2}$, the Ricci scalar, the Kretschmann scalar and the torsion scalar $T(r)$ as a function of $r$, for the positive and negative branches of black-hole solutions. The left graphs correspond to charged solutions $(Q=1)$, while the right graphs correspond to uncharged solutions $(Q=0)$.

The positive branch in the charged case exhibits only one physical singularity at $r=0$, in which the torsion scalar and the Ricci and Kretschmann scalars diverge, however it is shielded by two horizons at $r_{H}=r_{-}$and $r_{H}=r_{+}$, since in this case $G(r)^{2}$ has two roots. In order to examine whether $r_{+}$is a Killing horizon we see that the timelike Killing vector of the metric is $\epsilon^{\mu} \partial_{\mu}=\partial_{t}{ }^{1}$ with norm $\epsilon_{\mu} \epsilon^{\mu}=g_{t t}=F(r)^{2}$ which vanishes at $r=r_{+}$. Inside the horizon the Killing vector field is spacelike, while outside it is timelike, and thus it corresponds to a null hypersurface. Finally, for the uncharged solutions we can see that the Ricci scalar and the torsion scalar are constants, while the Kretschmann scalar diverge at $r=0$ (similarly to usual General Relativity).

For the negative branch in the charged case, the torsion scalar possesses only one divergence, namely at $r=0$, however the Ricci and Kretschmann scalars possess two divergence points, namely at $r=0$ and $r=r_{s}$ as described above. However, in this specific numerical example, both these physical singularities are shielded by the horizon at $r_{H}>r_{s}$, in which all invariants remain regular. In particular, $r_{H}$ is a Killing horizon, corresponding to an event horizon since the Killing vector field is timelike outside the horizon and spacelike inside. Finally, for the uncharged solutions the Ricci and torsion scalars are constants, but the Kretschmann scalar diverges at $r=0$. However, this physical singularity is shielded by the horizon at $r=r_{H}$ in which $G(r)^{2}$ becomes zero.

In summary, we indeed verify that in the charged case and for the negative branch the curvature invariants contain an extra divergence at $r=r_{s}$, that does not appear in the torsion invariant, revealing the novel features of charged $f(T)$ gravity.

- Case $\Lambda_{\text {eff }}>0$

We consider $\Lambda=1 / 25$, which satisfies the condition $\Lambda_{\text {eff }}>0$ for the positive branch (as we mentioned below (5.9) the negative branch cannot lead to $\Lambda_{\text {eff }}>0$ ). In figure 2 from up to down we depict $G(r)^{2}$, the Ricci scalar, the Kretschmann scalar and the torsion scalar $T(r)$ as a function of $r$, for the positive branch of black-hole solutions. The left graphs correspond to charged solutions $(Q=1)$, while the right graphs correspond to uncharged solutions $(Q=0)$.

The charged case possesses a physical singularity at $r=0$, where the torsion scalar and the Ricci and Kretschmann scalars diverge, however it is shielded a horizon

\footnotetext{
${ }^{1}$ Note that none of the metric coefficients depends on time and thus the manifold has a timelike Killing vector $\partial_{t}$, and similarly since none of the metric coefficients depends on $x_{i}$ there exist $(D-2)$ spacelike Killing vector fields $\partial_{x_{i}}$.
} 

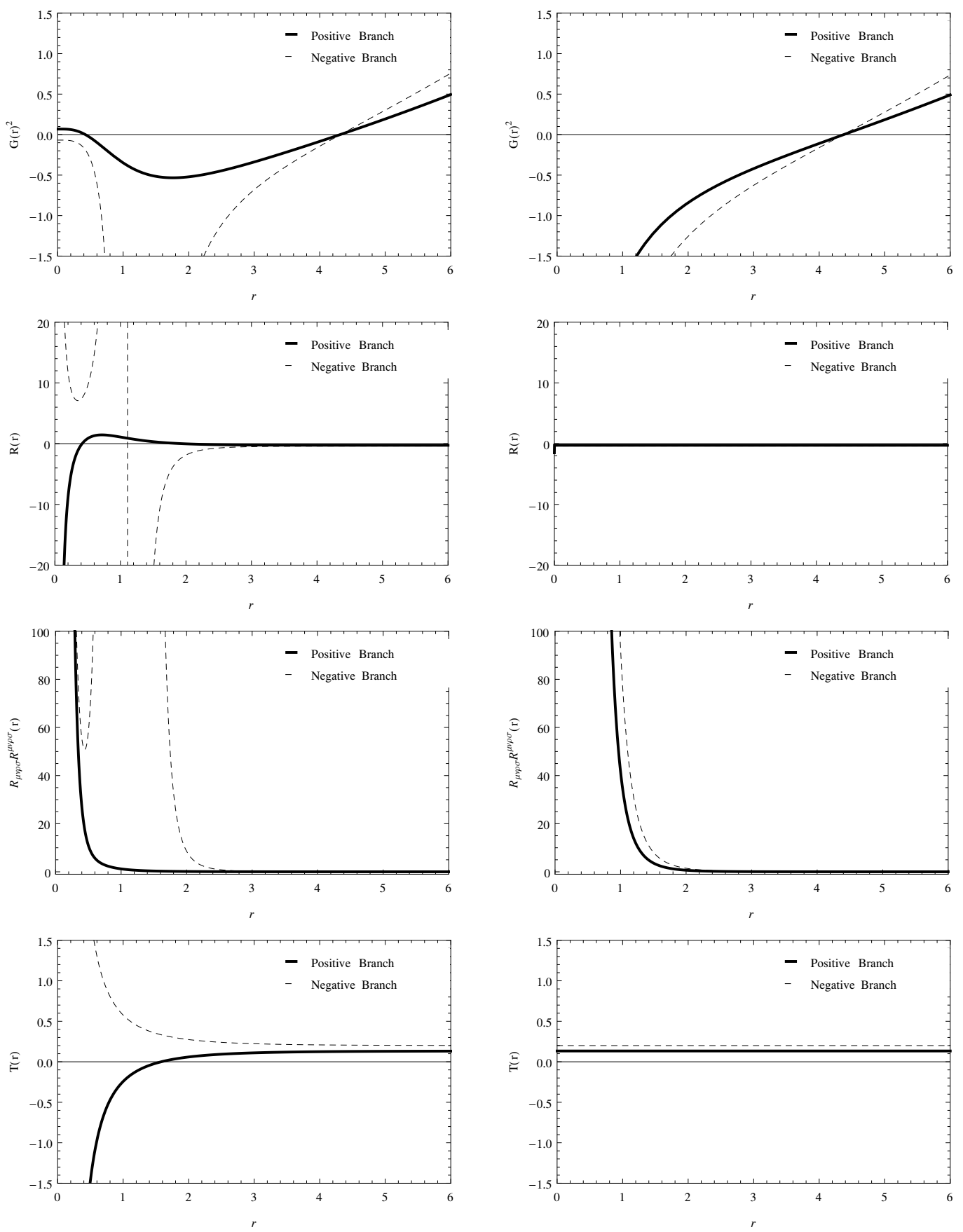

Figure 1. The solutions for $G(r)^{2}$ of (4.13), for the Ricci scalar $R(r)$ of (5.1), for the Kretschmann scalar $R_{\mu \nu \rho \sigma} R^{\mu \nu \rho \sigma}(r)$ of (5.3) and for the torsion scalar $T(r)$ of (4.9), as a function of $r$, for the positive (thick solid curve) and negative (thin dashed curve) branch of the AdS solution subclass, for $D=4, \alpha=-1$, Const $=-1$ and $\Lambda=-1 / 25$. Left graphs correspond to charged solutions with $Q=1$, while right graphs correspond to uncharged solutions with $Q=0$. The thin 0 -line is depicted for convenience. 

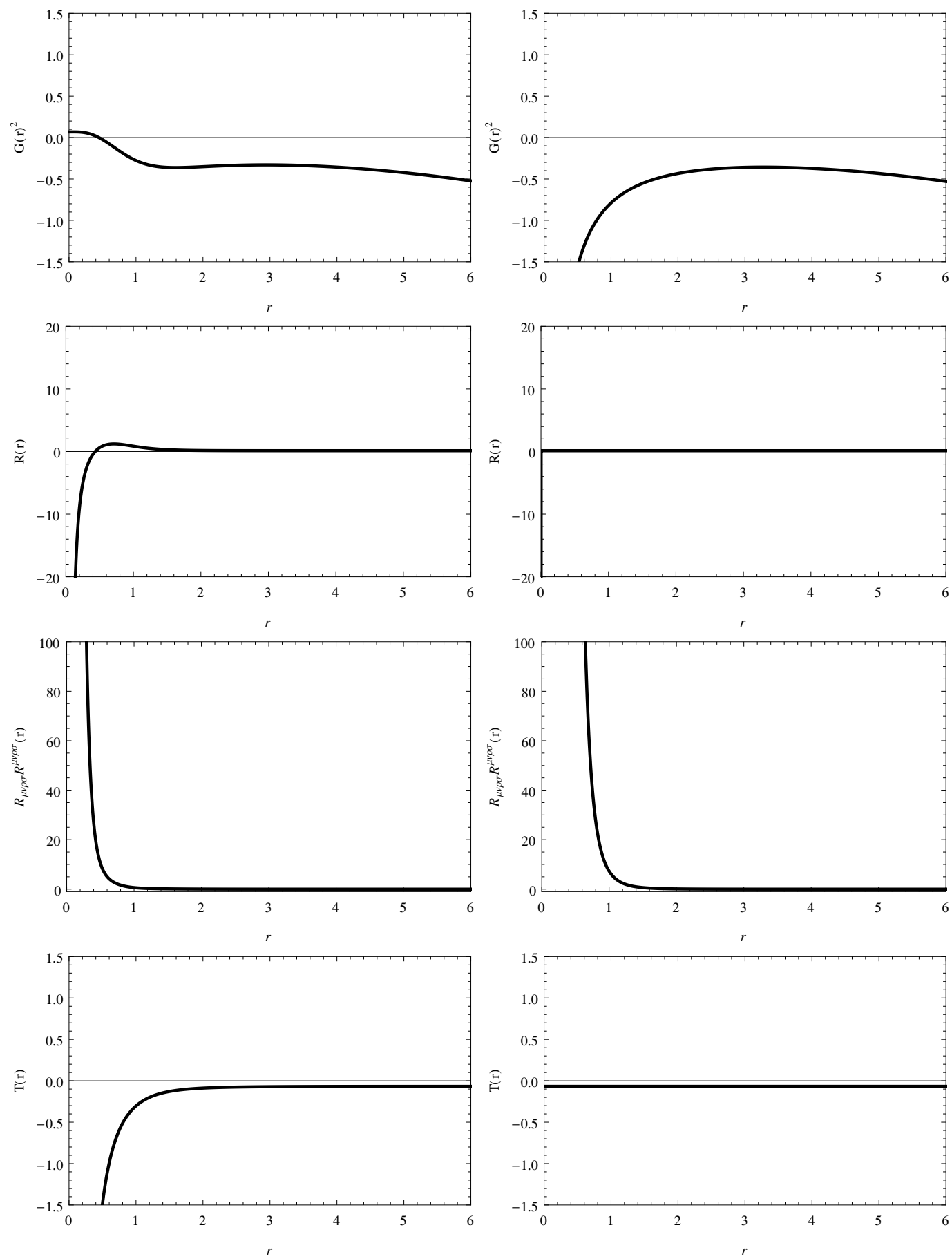

Figure 2. The solutions for $G(r)^{2}$ of (4.13), for the Ricci scalar $R(r)$ of (5.1), for the Kretschmann scalar $R_{\mu \nu \rho \sigma} R^{\mu \nu \rho \sigma}(r)$ of (5.3) and for the torsion scalar $T(r)$ of (4.9), as a function of $r$, for the positive (thick solid curve) branch of the dS solution subclass, for $D=4, \alpha=-1$, Const $=-1$ and $\Lambda=1 / 25$. Left graphs correspond to charged solutions with $Q=1$, while right graphs correspond to uncharged solutions with $Q=0$. The thin 0-line is depicted for convenience. 
at $r=r_{H}$, where $G(r)^{2}$ becomes zero. In order to examine whether $r_{H}$ is a Killing horizon, and similarly to the previous case, we observe that the timelike Killing vector of the metric is $\epsilon^{\mu} \partial_{\mu}=\partial_{t}$, with norm $\epsilon_{\mu} \epsilon^{\mu}=g_{t t}=F(r)^{2}$ which vanishes at $r=r_{H}$. Since outside the horizon the Killing vector field is timelike, and inside it is spacelike, it is implied that it corresponds to a null hypersurface, that is a cosmological Killing horizon.

In the uncharged case we observe that the Ricci scalar vanishes while the torsion scalar is constant, however the Kretschmann scalar diverges at $r=0$. However, note that in this case $G(r)^{2}$ has no roots, that is there is not a horizon to shield the physical singularity at $r=0$, which is therefore a naked one.

- Case $\Lambda_{\text {eff }}=0$

We consider $\Lambda=0$, which satisfies the condition $\Lambda_{\text {eff }}=0$ for the positive branch (as we mentioned below (5.9) the negative branch cannot lead to $\Lambda_{\text {eff }}=0$ ). In figure 3 from up to down we show $G(r)^{2}$, the Ricci scalar, the Kretschmann scalar and the torsion scalar $T(r)$ as a function of $r$, for the positive branch, with left graphs corresponding to charged solutions $(Q=1)$ and right graphs corresponding to uncharged solutions $(Q=0)$.

The charged solutions possess a physical singularity at $r=0$, where all invariants diverge, however it is shielded a horizon at $r=r_{H}$, where $G(r)^{2}$ becomes zero. Examining the Killing vector, and similarly to the previous cases, we deduce that $r_{H}$ is a cosmological Killing horizon. In the uncharged case we observe that both the Ricci and torsion scalars vanish, however the Kretschmann scalar diverges at $r=0$. Notice that in this case $G(r)^{2}$ has no roots, that is there is not a horizon to shield the physical singularity at $r=0$, which is therefore a naked one.

\section{Concluding remarks}

In this work we considered D-dimensional $f(T)$ gravity including the Maxwell field. We extracted exact charged black-hole solutions depending on the functional form of $f(T)$, on the electric charge and on the number the dimensionality D. Finally, we investigated the singularities and the horizons of the obtained solutions, following two different approaches. Firstly, by studying the torsion invariants constructed using the Weitzenböck's connection and the vierbein solutions, and secondly by studying the curvature invariants constructed using the Levi-Civita connection and the metric solutions.

The main result is that in Maxwell- $f(T)$ gravity the curvature invariants possess more physical singularities than the torsion ones, in some particular solution subclasses. This difference disappears in the uncharged case, or in the case where $f(T)$ gravity becomes the usual linear-in- $T$ teleparallel gravity, thus it reveals the novel behavior that is introduced by the combined complication of the non-trivial $f(T)$ structure with the electromagnetic sector. It seems that curvature and torsion invariants behave very differently depending on the presence of the Maxwell field. More generally, extending gravity in terms of $f(T)$ or $f(R)$ formulations could give very different results as soon as matter fields are taken into account. 

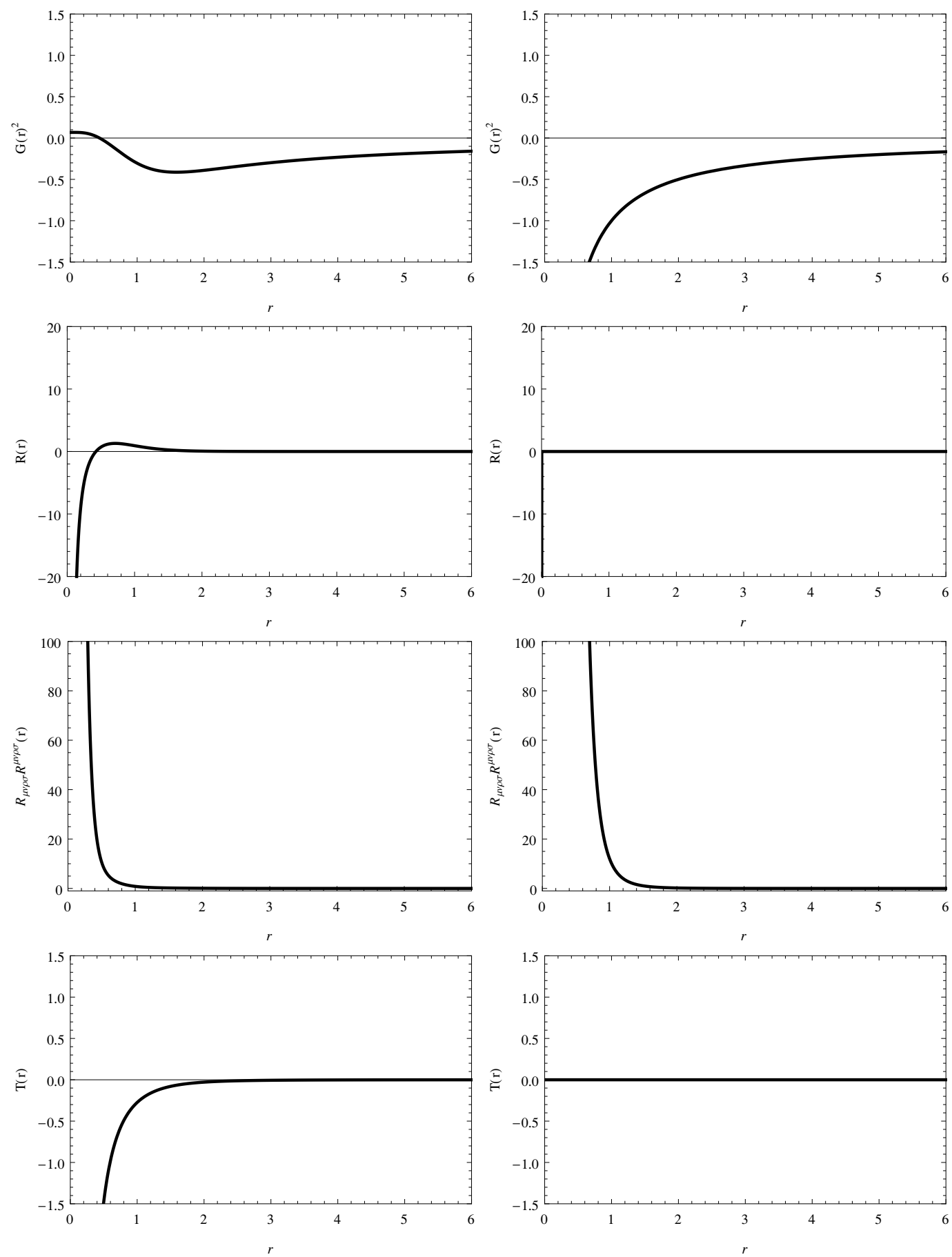

Figure 3. The solutions for $G(r)^{2}$ of (4.13), for the Ricci scalar $R(r)$ of (5.1), for the Kretschmann scalar $R_{\mu \nu \rho \sigma} R^{\mu \nu \rho \sigma}(r)$ of (5.3) and for the torsion scalar $T(r)$ of (4.9), as a function of $r$, for the positive (thick solid curve) branch, for $D=4, \alpha=-1$, Const $=-1$ and $\Lambda=0$. Left graphs correspond to charged solutions with $Q=1$, while right graphs correspond to uncharged solutions with $Q=0$. The thin 0 -line is depicted for convenience. 
Finally, we have to note that, in the scenario we have considered, the physical singularities are not always shielded by horizons. Thus, the cosmic censorship does not always hold for D-dimensional Maxwell- $f(T)$ gravity. From a cosmological point of view such a feature could be extremely relevant in order to investigate the early phases of cosmic evolution. On the other hand, considering astrophysical structures in strong field regimes, derived from torsion or curvature representation of gravity, could give rise to very deep differences in dynamics $[23,79]$.

\section{Acknowledgments}

S.C. is supported by INFN (Sez. di Napoli). The research of E.N.S. is implemented within the framework of the Action Supporting Postdoctoral Researchers of the Operational Program "Education and Lifelong Learning" (Actions Beneficiary: general Secretariat for Research and Technology), and is co-financed by the European Social Fund (ESF) and the Greek State. Y. V. is supported by FONDECYT grant 11121148, and by Dirección de Investigación y Desarrollo, Universidad de La Frontera, DIUFRO DI11-0071.

Open Access. This article is distributed under the terms of the Creative Commons Attribution License which permits any use, distribution and reproduction in any medium, provided the original author(s) and source are credited.

\section{References}

[1] A. Unzicker and T. Case, Translation of Einstein's attempt of a unified field theory with teleparallelism, physics/0503046 [INSPIRE].

[2] K. Hayashi and T. Shirafuji, New General Relativity, Phys. Rev. D 19 (1979) 3524 [Addendum ibid. D 24 (1982) 3312] [INSPIRE].

[3] R. Ferraro and F. Fiorini, Modified teleparallel gravity: Inflation without inflaton, Phys. Rev. D 75 (2007) 084031 [gr-qc/0610067] [INSPIRE].

[4] R. Ferraro and F. Fiorini, On Born-Infeld Gravity in Weitzenbock spacetime, Phys. Rev. D 78 (2008) 124019 [arXiv:0812.1981] [InSPIRE].

[5] G.R. Bengochea and R. Ferraro, Dark torsion as the cosmic speed-up, Phys. Rev. D 79 (2009) 124019 [arXiv:0812.1205] [INSPIRE].

[6] E.V. Linder, Einstein's Other Gravity and the Acceleration of the Universe, Phys. Rev. D 81 (2010) 127301 [Erratum ibid. D 82 (2010) 109902] [arXiv:1005.3039] [INSPIRE].

[7] R. Myrzakulov, Accelerating universe from F(T) gravity, Eur. Phys. J. C 71 (2011) 1752 [arXiv:1006.1120] [INSPIRE].

[8] S.-H. Chen, J.B. Dent, S. Dutta and E.N. Saridakis, Cosmological perturbations in $f(T)$ gravity, Phys. Rev. D 83 (2011) 023508 [arXiv: 1008.1250] [INSPIRE].

[9] P. Wu and H.W. Yu, $f(T)$ models with phantom divide line crossing, Eur. Phys. J. C 71 (2011) 1552 [arXiv:1008.3669] [InSPIRE]. 
[10] J.B. Dent, S. Dutta and E.N. Saridakis, $f(T)$ gravity mimicking dynamical dark energy. Background and perturbation analysis, JCAP 01 (2011) 009 [arXiv:1010.2215] [INSPIRE].

[11] R. Zheng and Q.-G. Huang, Growth factor in $f(T)$ gravity, JCAP 03 (2011) 002 [arXiv: 1010.3512] [INSPIRE].

[12] K. Bamba, C.-Q. Geng, C.-C. Lee and L.-W. Luo, Equation of state for dark energy in $f(T)$ gravity, JCAP 01 (2011) 021 [arXiv: 1011.0508] [INSPIRE].

[13] R.-J. Yang, Conformal transformation in $f(T)$ theories, Europhys. Lett. 93 (2011) 60001 [arXiv: 1010.1376] [INSPIRE].

[14] P. Wu and H.W. Yu, Observational constraints on $f(T)$ theory, Phys. Lett. B 693 (2010) 415 [arXiv:1006.0674] [INSPIRE].

[15] G.R. Bengochea, Observational information for $f(T)$ theories and Dark Torsion, Phys. Lett. B 695 (2011) 405 [arXiv:1008.3188] [InSPIRE].

[16] P. Wu and H.W. Yu, The dynamical behavior of $f(T)$ theory, Phys. Lett. B 692 (2010) 176 [arXiv: 1007.2348] [INSPIRE].

[17] Y. Zhang, H. Li, Y. Gong and Z.-H. Zhu, Notes on $f(T)$ Theories, JCAP 07 (2011) 015 [arXiv: 1103.0719] [INSPIRE].

[18] B. Li, T.P. Sotiriou and J.D. Barrow, Large-scale Structure in $f(T)$ Gravity, Phys. Rev. D 83 (2011) 104017 [arXiv:1103.2786] [INSPIRE].

[19] Y.-F. Cai, S.-H. Chen, J.B. Dent, S. Dutta and E.N. Saridakis, Matter Bounce Cosmology with the $f(T)$ Gravity, Class. Quant. Grav. 28 (2011) 215011 [arXiv:1104.4349] [INSPIRE].

[20] S. Chattopadhyay and U. Debnath, Emergent universe in chameleon, $f(R)$ and $f(T)$ gravity theories, Int. J. Mod. Phys. D 20 (2011) 1135 [arXiv:1105.1091] [InSPIRE].

[21] M. Sharif and S. Rani, F(T) Models within Bianchi Type I Universe, Mod. Phys. Lett. A 26 (2011) 1657 [arXiv:1105.6228] [INSPIRE].

[22] H. Wei, X.-P. Ma and H.-Y. Qi, $f(T)$ Theories and Varying Fine Structure Constant, Phys. Lett. B 703 (2011) 74 [arXiv:1106.0102] [InSPIRE].

[23] C.G. Boehmer, A. Mussa and N. Tamanini, Existence of relativistic stars in $f(T)$ gravity, Class. Quant. Grav. 28 (2011) 245020 [arXiv:1107.4455] [INSPIRE].

[24] H. Wei, H.-Y. Qi and X.-P. Ma, Constraining $f(T)$ Theories with the Varying Gravitational Constant, Eur. Phys. J. C 72 (2012) 2117 [arXiv:1108.0859] [INSPIRE].

[25] S. Capozziello, V. Cardone, H. Farajollahi and A. Ravanpak, Cosmography in f(T)-gravity, Phys. Rev. D 84 (2011) 043527 [arXiv:1108.2789] [InSPIRE].

[26] M. Hamani Daouda, M.E. Rodrigues and M. Houndjo, Static Anisotropic Solutions in $f(T)$ Theory, Eur. Phys. J. C 72 (2012) 1890 [arXiv:1109.0528] [InSPIRE].

[27] K. Bamba and C.-Q. Geng, Thermodynamics of cosmological horizons in $f(T)$ gravity, JCAP 11 (2011) 008 [arXiv:1109.1694] [INSPIRE].

[28] C.-Q. Geng, C.-C. Lee, E.N. Saridakis and Y.-P. Wu, 'Teleparallel' Dark Energy, Phys. Lett. B 704 (2011) 384 [arXiv:1109.1092] [INSPIRE].

[29] H. Wei, Dynamics of Teleparallel Dark Energy, Phys. Lett. B 712 (2012) 430 [arXiv: 1109.6107] [INSPIRE]. 
[30] C.-Q. Geng, C.-C. Lee and E.N. Saridakis, Observational Constraints on Teleparallel Dark Energy, JCAP 01 (2012) 002 [arXiv:1110.0913] [INSPIRE].

[31] C.G. Boehmer, T. Harko and F.S. Lobo, Wormhole geometries in modified teleparralel gravity and the energy conditions, Phys. Rev. D 85 (2012) 044033 [arXiv:1110.5756] [INSPIRE].

[32] J. Yang, Y.-L. Li, Y. Zhong and Y. Li, Thick Brane Split Caused by Spacetime Torsion, Phys. Rev. D 85 (2012) 084033 [arXiv:1202.0129] [InSPIRE].

[33] C. Xu, E.N. Saridakis and G. Leon, Phase-Space analysis of Teleparallel Dark Energy, JCAP 07 (2012) 005 [arXiv: 1202.3781] [INSPIRE].

[34] K. Bamba, R. Myrzakulov, S. Nojiri and S.D. Odintsov, Reconstruction of $f(T)$ gravity: Rip cosmology, finite-time future singularities and thermodynamics,

Phys. Rev. D 85 (2012) 104036 [arXiv:1202.4057] [InSPIRE].

[35] M. Setare and M. Houndjo, Finite-time future singularities models in $f(T)$ gravity and the effects of viscosity, arXiv:1203.1315 [INSPIRE].

[36] D. Liu, P. Wu and H. Yu, Gódel-type universes in $f(T)$ gravity, Int. J. Mod. Phys. D 21 (2012) 1250074 [arXiv:1203.2016] [InSPIRE].

[37] L. Iorio and E.N. Saridakis, Solar system constraints on $f(T)$ gravity, arXiv:1203.5781 [INSPIRE].

[38] H. Dong, Y.-b. Wang and X.-h. Meng, Extended Birkhoff's Theorem in the $f(T)$ Gravity, Eur. Phys. J. C 72 (2012) 2002 [arXiv:1203.5890] [INSPIRE].

[39] M.E. Rodrigues, M.H. Daouda and M. Houndjo, Inhomogeneous Universe in $f(T)$ Theory, arXiv: 1205.0565 [INSPIRE].

[40] K. Bamba, S. Capozziello, S. Nojiri and S.D. Odintsov, Dark energy cosmology: the equivalent description via different theoretical models and cosmography tests, Astrophys. Space Sci. 342 (2012) 155 [arXiv:1205.3421] [INSPIRE].

[41] D. Liu and M. Reboucas, Energy conditions bounds on $f(T)$ gravity, Phys. Rev. D 86 (2012) 083515 [arXiv:1207.1503] [INSPIRE].

[42] M. Wanas and H. Hassan, Torsion and Problems of Standard Cosmology, arXiv:1209.6218 [INSPIRE].

[43] F. Rahaman, R. Biswas, H.I. Fatima and N. Islam, A new proposal for Galactic dark matter: Effect of $f(T)$ gravity, arXiv:1207.2145 [INSPIRE].

[44] R. Ghosh and S. Chattopadhyay, Generalized second law of thermodynamics in the emergent universe for some viable models of $f(T)$ gravity, arXiv:1207.6024 [INSPIRE].

[45] M. Rodrigues, M. Houndjo, D. Saez-Gomez and F. Rahaman, Anisotropic Universe Models in $f(T)$ Gravity, Phys. Rev. D 86 (2012) 104059 [arXiv:1209.4859] [InSPIRE].

[46] S. Capozziello and M. De Laurentis, Extended Theories of Gravity, Phys. Rept. 509 (2011) 167 [arXiv:1108.6266] [INSPIRE].

[47] S. Capozziello and M. Francaviglia, Extended Theories of Gravity and their Cosmological and Astrophysical Applications, Gen. Rel. Grav. 40 (2008) 357 [arXiv:0706.1146] [INSPIRE].

[48] M. Bañados, C. Teitelboim and J. Zanelli, The Black hole in three-dimensional space-time, Phys. Rev. Lett. 69 (1992) 1849 [hep-th/9204099] [INSPIRE]. 
[49] S. Capozziello and V. Faraoni, Beyond Einstein Gravity: A Survey of gravitational theories for cosmology and astrophysics, Fund. Theor. 170 (2011). [INSPIRE]

[50] Weitzenböck R., Invarianten Theorie, Nordhoff, Groningen, The Netherlands (1923).

[51] S. Capozziello, G. Lambiase and C. Stornaiolo, Geometric classification of the torsion tensor in space-time, Annalen Phys. 10 (2001) 713 [gr-qc/0101038] [INSPIRE].

[52] J. Maluf, Hamiltonian formulation of the teleparallel description of general relativity, J. Math. Phys. 35 (1994) 335 [inSPIRE].

[53] H. Arcos and J. Pereira, Torsion gravity: A Reappraisal, Int. J. Mod. Phys. D 13 (2004) 2193 [gr-qc/0501017] [inSPIRE].

[54] S. Weinberg, Cosmology, Oxford University Press, Oxford, U.K. (2008).

[55] B. Li, T.P. Sotiriou and J.D. Barrow, $f(T)$ gravity and local Lorentz invariance, Phys. Rev. D 83 (2011) 064035 [arXiv:1010.1041] [INSPIRE].

[56] T.P. Sotiriou, B. Li and J.D. Barrow, Generalizations of teleparallel gravity and local Lorentz symmetry, Phys. Rev. D 83 (2011) 104030 [arXiv: 1012.4039] [INSPIRE].

[57] M. Li, R.-X. Miao and Y.-G. Miao, Degrees of freedom of $f(T)$ gravity, JHEP 07 (2011) 108 [arXiv:1105.5934] [INSPIRE].

[58] U. Muench, F. Gronwald and F.W. Hehl, A Small guide to variations in teleparallel gauge theories of gravity and the Kaniel-Itin model, Gen. Rel. Grav. 30 (1998) 933 [gr-qc/9801036] [INSPIRE].

[59] Y. Itin, Coframe teleparallel models of gravity: Exact solutions, Int. J. Mod. Phys. D 10 (2001) 547 [gr-qc/9912013] [INSPIRE].

[60] R. Ferraro and F. Fiorini, Non trivial frames for $f(T)$ theories of gravity and beyond, Phys. Lett. B 702 (2011) 75 [arXiv:1103.0824] [INSPIRE].

[61] R. Ferraro and F. Fiorini, Cosmological frames for theories with absolute parallelism, Int. J. Mod. Phys. Conf. Ser. 3 (2011) 227 [arXiv:1106.6349] [inSPIRE].

[62] N. Tamanini and C.G. Boehmer, Good and bad tetrads in $f(T)$ gravity, Phys. Rev. D 86 (2012) 044009 [arXiv:1204.4593] [INSPIRE].

[63] M.H. Daouda, M.E. Rodrigues and M. Houndjo, Anisotropic fluid for a set of non-diagonal tetrads in $f(T)$ gravity, Phys. Lett. B 715 (2012) 241 [arXiv:1202.1147] [INSPIRE].

[64] T. Wang, Static Solutions with Spherical Symmetry in $f(T)$ Theories, Phys. Rev. D 84 (2011) 024042 [arXiv:1102.4410] [INSPIRE].

[65] R.-X. Miao, M. Li and Y.-G. Miao, Violation of the first law of black hole thermodynamics in $f(T)$ gravity, JCAP 11 (2011) 033 [arXiv:1107.0515] [INSPIRE].

[66] H. Wei, X.-J. Guo and L.-F. Wang, Noether Symmetry in $f(T)$ Theory, Phys. Lett. B 707 (2012) 298 [arXiv:1112.2270] [INSPIRE].

[67] R. Ferraro and F. Fiorini, Spherically symmetric static spacetimes in vacuum $f(T)$ gravity, Phys. Rev. D 84 (2011) 083518 [arXiv:1109.4209] [INSPIRE].

[68] P. Gonzalez, E.N. Saridakis and Y. Vasquez, Circularly symmetric solutions in three-dimensional Teleparallel, $f(T)$ and Maxwell- $f(T)$ gravity, JHEP 07 (2012) 053 [arXiv:1110.4024] [INSPIRE]. 
[69] M. Cataldo, Azimuthal electric field in a static rotationally symmetric $(2+1)$-dimensional space-time, Phys. Lett. B 529 (2002) 143 [gr-qc/0201047] [inSPIRE].

[70] M. Blagojevic and B. Cvetkovic, Electric field in 3D gravity with torsion, Phys. Rev. D 78 (2008) 044036 [arXiv:0804.1899] [INSPIRE].

[71] C. Pun, Z. Kovacs and T. Harko, Thin accretion disks in $f(R)$ modified gravity models, Phys. Rev. D 78 (2008) 024043 [arXiv:0806.0679] [InSPIRE].

[72] A. de la Cruz-Dombriz, A. Dobado and A. Maroto, Black Holes in $f(R)$ theories, Phys. Rev. D 80 (2009) 124011 [Erratum ibid. D 83 (2011) 029903] [arXiv:0907.3872] [INSPIRE].

[73] S.E. Perez Bergliaffa and Y.E.C. de Oliveira Nunes, Static and spherically symmetric black holes in $f(R)$ theories, Phys. Rev. D 84 (2011) 084006 [arXiv:1107.5727] [InSPIRE].

[74] S.H. Mazharimousavi and M. Halilsoy, Comment on 'Static and spherically symmetric black holes in $f(R)$ theories', Phys. Rev. D 86 (2012) 088501 [arXiv:1210.4699] [InSPIRE].

[75] P. Painlevé, La Mécanique classique et la théorie de la relativité, C. R. Acad. Sci. (Paris) 173 (1921) 677.

[76] A. Gullstrand, Allegemeine Lösung des statischen Einkörper-problems in der Einsteinschen Gravitations theorie, Arkiv. Mat. Astron. Fys. 16 (1922) 1.

[77] K. Martel and E. Poisson, Regular coordinate systems for Schwarzschild and other spherical space-times, Am. J. Phys. 69 (2001) 476 [gr-qc/0001069] [INSPIRE].

[78] W. Liu, New coordinates for BTZ black hole and Hawking radiation via tunnelling, Phys. Lett. B 634 (2006) 541 [gr-qc/0512099] [INSPIRE].

[79] S. Capozziello, M. De Laurentis, S. Odintsov and A. Stabile, Hydrostatic equilibrium and stellar structure in $f(R)$-gravity, Phys. Rev. D 83 (2011) 064004 [arXiv:1101.0219] [INSPIRE]. 